\title{
EMISSION LINE REGIONS IN M86
}

\author{
S. DÖBEREINER ${ }^{1}$, J. HEIDT ${ }^{2}$, S. J. WAGNER, ${ }^{2}$ \\ ${ }^{1}$ Max-Planck Institut für Extraterrestrische Physik, Garching, \\ Germany; ${ }^{2}$ Landessternwarte Heidelberg, Germany
}
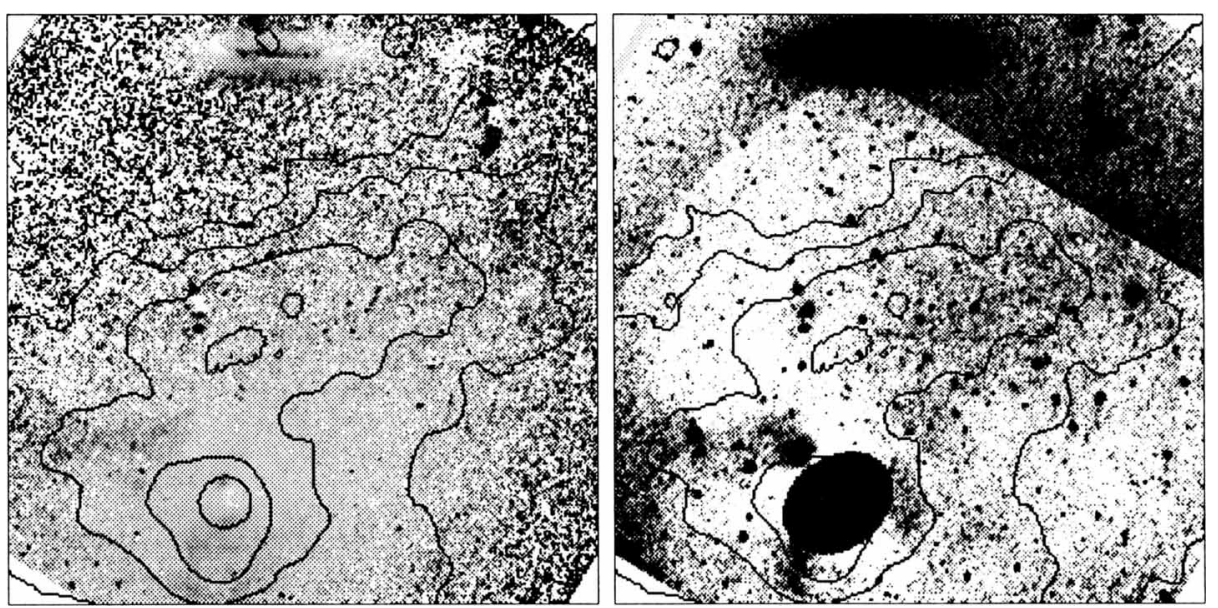

We present broad- and narrow-band $(\mathrm{B}, \mathrm{R}, \mathrm{H} \alpha+[\mathrm{NII}]$ and $\mathrm{H} \alpha$ contınuum) observations of M86 obtained with a focal reducer at the Calar Alto $1.23 \mathrm{~m}$ telescope, to look for any signs of material cooling out from the hot X-ray emitting ISM. The above figures show a quotient image (13' $\mathrm{x}$ 13', north up) ( $\mathrm{H} \alpha$ cont. - $\mathrm{H} \alpha$ ) to the left, and a $\mathrm{H} \alpha$ image (right) from which the light of the stellar spheroid of M86 has been subtracted. Both are overlayed with ROSAT PSPC X-ray contours.

We found three regions of excess emission (dark in the figures): (A): $\mathrm{H} \alpha$ emission with complex morphology near and south of the center of M86, possibly related to cooling flow activity. (B): A broad fanshaped feature extending to the $\mathrm{NE}$ and $\mathrm{E}$, visible as excess in the galaxy-subtracted images of all bands, although most prominent in the $\mathrm{H} \alpha$ band. The head of this feature is coincident with a small companion galaxy, so it is probably due to interaction. (C): A very narrow filament extending along the X-ray plume to the northwest. This filament may be due to cooling gas in the denser regions of the X-ray plume or to shock heating of yet unknown origin. 